\title{
Impact of gonadectomy on maturational changes in brain volume in adolescent macaques
}

\author{
Rebecca C. Knickmeyer ${ }^{\mathrm{a}, \mathrm{b}, *}$, Crystal T. Nguyen ${ }^{\mathrm{c}}$, Jeffrey T. Young ${ }^{\mathrm{b}}$, Anne Haunton ${ }^{\mathrm{d}, 1}$, \\ Michael R. Kosorok ${ }^{\mathrm{c}}$, John H. Gilmore ${ }^{\mathrm{b}}$, Martin Styner ${ }^{\mathrm{b}, \mathrm{e}}$, Debora A. Rothmond ${ }^{\mathrm{f}}$, \\ Pamela L. Noble ${ }^{g}$, Rhoshel Lenroot ${ }^{\mathrm{h}}$, Cynthia Shannon Weickert ${ }^{\mathrm{i}, \mathrm{j}}$
}

\footnotetext{
${ }^{a}$ Michigan State University, Institute for Quantitative Health Science and Engineering, Room 2114, Bio Engineering Facility, 775 Woodlot Dr., East Lansing, MI 48824, USA

${ }^{\mathrm{b}}$ University of North Carolina at Chapel Hill, Department of Psychiatry, Campus Box \#7160, Chapel Hill, NC 27599-7160, USA

${ }^{\mathrm{c}}$ University of North Carolina at Chapel Hill, Department of Biostatistics, Campus Box \#7420, Chapel Hill, NC 27599-7420, USA

${ }^{\mathrm{d}}$ North Carolina School of Science and Mathematics, 1219 Broad St, Durham, NC 27705, USA

${ }^{\mathrm{e}}$ University of North Carolina at Chapel Hill, Department of Computer Science, Campus Box \#3175, Chapel Hill, NC 27599-3175, USA

${ }^{\mathrm{f}}$ Schizophrenia Research Laboratory, Neuroscience Research Australia, Randwick, NSW 2031, Australia

${ }^{g}$ Laboratory of Neuropsychology, National Institute for Mental Health, National Institutes of Health, Bethesda, MD 20892-9663, USA

${ }^{\mathrm{h}}$ University of New Mexico, Department of Psychiatry and Behavioral Sciences, Albuquerque, NM 87131, USA

${ }^{\mathrm{i}}$ School of Psychiatry, Faculty of Medicine, University of New South Wales, Sydney, NSW 2052, Australia

${ }^{\mathrm{j}}$ Department of Neuroscience \& Physiology, Upstate Medical University, Syracuse, NY 13210, USA
}

\section{A R T I C L E I N F O}

Keywords:

Macaca mulatta

Adolescent

Prefrontal cortex

Magnetic resonance imaging

Testosterone

\section{A B S T R A C T}

Adolescence is a transitional period between childhood and adulthood characterized by significant changes in global and regional brain tissue volumes. It is also a period of increasing vulnerability to psychiatric illness. The relationship between these patterns and increased levels of circulating sex steroids during adolescence remains unclear. The objective of the current study was to determine whether gonadectomy, prior to puberty, alters adolescent brain development in male rhesus macaques. Ninety-six structural MRI scans were acquired from 12 male rhesus macaques ( 8 time points per animal over a two-year period). Six animals underwent gonadectomy and 6 animals underwent a sham operation at 29 months of age. Mixed-effects models were used to determine whether gonadectomy altered developmental trajectories of global and regional brain tissue volumes. We observed a significant effect of gonadectomy on the developmental trajectory of prefrontal gray matter (GM), with intact males showing peak volumes around 3.5 years of age with a subsequent decline. In contrast, prefrontal GM volumes continued to increase in gonadectomized males until the end of the study. We did not observe a significant effect of gonadectomy on prefrontal white matter or on any other global or regional brain tissue volumes, though we cannot rule out that effects might be detected in a larger sample. Results suggest that the prefrontal cortex is more vulnerable to gonadectomy than other brain regions.

\section{Introduction}

Adolescence is a distinct phase of development extending from pubertal onset to the emergence of adult appearance, physiology, and identity. Substantial changes in cognition and behavior throughout this period are accompanied by significant changes in brain maturation (Brenhouse and Andersen, 2011). In humans, cortical volume, measured via structural magnetic resonance imaging (sMRI), follows an inverted-U-shaped cubic trajectory between three and thirty years of age (Raznahan et al., 2011). It peaks in the peripubertal period (late

\footnotetext{
* Corresponding author at: Institute for Quantitative Health Science and Engineering, Room 2114, Bio Engineering Facility, 775 Woodlot Dr., East Lansing, MI 48824, USA.

E-mail addresses: knickmey@msu.edu (R.C. Knickmeyer), ctn92@live.unc.edu (C.T. Nguyen), jeffrey@unc.edu (J.T. Young), alhaunto@nscu.edu (A. Haunton),

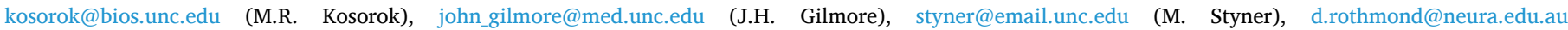
(D.A. Rothmond), noblep@mail.nih.gov (P.L. Noble), RLenroot@salud.unm.edu (R. Lenroot), c.weickert@neura.edu.au (C.S. Weickert).

1 Currently North Carolina State University, Raleigh, NC 27695, USA.
} 
childhood, early adolescence) and then reduces with decreasing velocity until itstabilizes around twenty years of age. This overall pattern reflects changes in both gray matter (GM), which exhibits a nonlinear pattern of development (pre-pubertal increases followed by adolescent decreases), and white matter (WM), which exhibits a relatively linear increase from childhood to young adulthood (Aubert-Broche et al., 2013; Giedd et al., 1999). Significant regional heterogeneity is present. For example, parietal gray matter (GM) shows marked reductions in volume which begin prior to puberty (perhaps as early as five years of age) and continue throughout adolescence, although the rate of change attenuates with increasing age (Aubert-Broche et al., 2013; Tamnes et al., 2013; Vijayakumar et al., 2016). In contrast, anterior regions appear to reach peak volumes in the peripubertal period, and display accelerating volume reductions across adolescence (Aubert-Broche et al., 2013; Giedd, 2004; Tamnes et al., 2013; Vijayakumar et al., 2016).

Sex differences have also been reported at both the global and regional level (Aubert-Broche et al., 2013; Dennison et al., 2013; Lenroot et al., 2007; Mills et al., 2016; Raznahan et al., 2011; Vijayakumar et al., 2016). Females achieve peak cortical volumes earlier (around 8 years) compared to males (9.3 years) (Raznahan et al., 2011) and males demonstrate protracted increases in WM across adolescence compared to females (Aubert-Broche et al., 2013). Information on regional sex differences is somewhat inconsistent. However, data from the Philadelphia Neurodevelopmental Cohort suggests that there are sex differences in hippocampal development with pre-pubertal males and females exhibiting similar hippocampal volumes (relative to intracranial volume), but post-pubertal females exhibiting larger hippocampal volumes than males (Satterthwaite et al., 2014). Males also show greater brain volume variance than females across multiple structures, an effect which is relatively stable throughout childhood and adolescence (Weirenga et al., 2017). For a more comprehensive review readers are directed to Kaczkurkin et al. (2019).

Unfortunately, the period of adolescent brain maturation is also a period of increased vulnerability to psychopathology (Mendle, 2014). During adolescence, depression, anxiety, and eating disorders increase in females (Angold et al., 2003; Hayward and Sanborn, 2002), conduct problems and aggression rise for both genders (Mendle, 2014), and onset of schizophrenia is more common in males (Gotowiec et al., 2003).

It is currently unclear how these developmental brain changes relate to the substantial increases in primary sex steroids that accompany puberty. Several studies of human adolescents have reported associations between circulating sex steroid levels and GM and WM volumes (Bramen et al., 2011; Herting et al., 2014; Neufang et al., 2009; Peper et al., 2009), but the pattern of results is inconsistent, perhaps due to heterogeneity in design, subject demographics, and approaches to image acquisition and analysis. Furthermore, these observational studies cannot distinguish between the possibility that circulating hormones play a causal role in shaping adolescent brain development and the possibility that circulating hormone levels are serving as a proxy for some other causal factor. Well-controlled, experimental studies are necessary to overcome these limitations.

Experimental studies in rodents indicate that gonadal hormones influence sex differences in the adult brain (for review see Schulz and Sisk (2016) and McEwen and Milner (2017)). For example, seminal work conducted by Bruce McEwen revealed that estrogen increases spine density in the hippocampus of adult female rats (reviewed in McEwen et al., 2012), while testosterone increases dendritic spine density in the CA1 region of the adult male rat hippocampus (Romeo et al., 2005). Gonadal steroids also play causal roles in the emergence of neurobiological sex differences during adolescence. For example, pubertal ovarian steroids appear to promote apoptosis in primary visual cortex (Nunez et al., 2001, 2002) and ventral mPFC of female rats (Koss et al., 2015), while pubertal testosterone influences regional volume and neuron number within the medial amygdala of adult male Syrian hamsters (De Lorme et al., 2012). In some cases, gonadal hormones may be necessary for maintaining neurobiological sex differences established in the prenatal and early postnatal period (Ahmed et al., 2008; Breedlove et al., 2002; Morishita et al., 2017). However, the relevance of these findings to humans is currently unclear due to significant species differences in pubertal physiology, brain development, and brain structure (Borrell and Calegari, 2014; Hodges and Palmert, 2007).

Compared to rodents, the rhesus macaque possesses a greater similarity to humans in terms of the extended time course of puberty and brain maturation, and in possessing a more convoluted cortical brain structure (Hodges and Palmert, 2007; Knickmeyer et al., 2010; Scott et al., 2016). Consequently, this species is especially informative as a translational model. Encephalization of the macaque cortex is also conducive to state-of-the-art neuroimaging (Young et al., 2017). Thus, the objective of the current study was to determine whether gonadectomy, prior to puberty, alters adolescent brain development in male rhesus macaques using sMRI. We have previously shown that gonadectomy prior to puberty increases hippocampal neurogenesis, leads to a drop in position in the social hierarchy, attenuates sensory-motor gating, and alters reactions to emotional faces of con-specifics during adolescence (Allen et al., 2014; Morris et al., 2010; Richards et al., 2009). How gonadectomy influences maturational trajectories of global, cortical, and subcortical brain volumes has not been previously reported. Based on prior research suggesting that pubertal testosterone closes the "growth" window or plasticity window for hippocampal neurogenesis (Allen et al., 2014), we hypothesized that gonadectomized monkeys would exhibit prolonged maturational trajectories compared to intact animals (e.g. extended growth).

\section{Materials and methods}

\subsection{Subjects}

Twelve experimentally naive male rhesus macaque monkeys (Macaca mulatta) from the NIH Animal Center's primate field station were used in this study. Detailed information on matriline and maternal rank, housing conditions, and feeding can be found in Richards et al. (2009). At the start of the study (27 months), all monkeys weighed between 3.2 and $5.39 \mathrm{~kg}$, had basal morning testosterone levels of $<0.06$ ng $\mathrm{mL}-1$, and none showed overt signs of puberty (Bowen and Koch, 1970). The sample size represents all eligible animals that could be acquired from the NIH Animal Center's primate field station in a two-year period, given constraints such as the size of the birth cohort, the number of males, and the number of other projects being conducted.

\subsection{Surgeries and subsequent assessments}

At 29 months of age, monkeys were randomly selected for either gonadectomy $(n=6)$ or sham operation $(n=6)$ with the constraint that resulting groups were comparable in average matriline rank, maternal rank, and body weight. Sham animals underwent the same anesthesia protocol but did not have any surgical manipulations. Mean (SD) of age at time of surgery was $29.18(0.44)$ months for sham animals and 29.09 (0.47) months for gonadectomized animals (test for group differences: $\mathrm{df}$ $=9.94, \mathrm{p}$-value $=0.73, \mathrm{t}=0.36$ ). The surgeries were performed in a yoked fashion on aged matched monkeys, where castration was performed on one animal and sham surgery was performed on the other animal by the same surgical team, with the same equipment, on the same day, with the same recovery period. Blood samples were collected every 6-8 weeks from 1 month prior to surgery until study conclusion in order to assess plasma levels of testosterone. Samples were taken at 9:30 am and 11:00 pm to account for diurnal variation. Plasma testosterone levels were measured by radioimmunoassay at the Yerkes National Primate Research Center Biomarkers Core Lab at Emory University. Additional details can be found in Richards et al. (2009).

For the acquisition of the MRIs, a General Electric 3 T MRI (voxel size: $0.43 \times 0.43 \times 0.8 \mathrm{~mm}^{3}$ ) scanner was utilized. The MRI image acquisition parameters were Field of View $=30 \mathrm{~mm}$, Repetition Time $=$ 
$9 \mathrm{~ms}$, Echo Time $=4 \mathrm{~ms}$ (minimum). As the monkey heads were too small for a human head coil, the subjects were scanned using a knee coil. All monkeys were anesthetized for the duration of the MRI assessment to reduce motion artefacts. Eight separate scans were acquired in each subject over a 2-year period. All procedures were approved by the National Institute of Mental Health (NIMH) Animal Care and Use Ethics Committee and comply with the National Research Council's guide for the care and use of laboratory animals and with the ARRIVE guidelines.

\subsection{Image analysis}

Longitudinal image processing frameworks (for example using the baseline scan as a reference for each subject or propagating measurements directly from one age to another age) can easily yield biased measurements (see also Reuter and Fischl (2011)). Consequently, we chose to employ an independent processing approach in this study. T1w scans were first corrected for intensity inhomogeneity via N4, followed by rigid alignment into the UNC-Emory 12-month atlas space (Shi et al., 2016) and automatic brain mask computation using AutoSeg (v3.3.2) (Wang et al., 2014). The automatic brain masks were all manually corrected for the purpose of skull stripping. Skull stripped T1w images were then processed via a standard atlas moderated Expectation Maximization tissue segmentation, single atlas subcortical segmentation, and regional parcellation (Knickmeyer et al., 2010) in AutoSeg. We employ an affine registration followed by a deformable registration (using the symmetric diffeomorphic registration in ANTS) in order to register each image to the relevant atlases. Regional WM parcellation volumes were calculated for the following regions: cerebellum, cingulate cortex, prefrontal cortex (PFC), frontal cortex, insular cortex, parietal lobe, occipital lobe, visual temporal cortex, limbic temporal cortex, auditory temporal cortex, combined pons and medulla, combined subcortical region, and the corpus callosum. Regional GM parcellation volumes were calculated for all the above regions except the corpus callosum. In addition to the combined subcortical region noted above, separate subcortical volumes were calculated for the putamen, hippocampus and caudate. We did not calculate separate volumes for the amygdala due to the challenges of reliably identifying the boundaries of the amygdala in our images. Visual inspection was performed to assess the quality of the segmentation using ITK-Snap (www.itksnap.org). Datasets failing visual inspection were excluded from the analysis (one out of 96 scans failed visual inspection). No manual correction of the automatically segmented data was performed. Individuals performing image analysis and quality control were blind to experimental condition (gonadectomized versus intact). See Supplementary Fig. 1 for an example of a successful segmentation.

\subsection{Statistical analysis}

In order to determine whether gonadectomy successfully eliminated the expected increase in adolescent testosterone, linear mixed effects models with random intercepts were fit to testosterone values using time (age), condition, and time of day as covariates, including a time by condition interaction. Time of day indicates whether the measurement was taken in the morning (time of day $=1$ ) or in the evening (time of day $=0$ ). In intact animals, testosterone exhibits a clear diurnal pattern (lower values in the morning, higher values in the evening). Values of testosterone below the clinical level of detection $(0.05 \mu \mathrm{g} / \mathrm{mL})$ were fixed at 0.05 for analysis. Diagnostics for the initial model revealed skewed residuals, indicating a violation of the linearity assumption necessary for a valid linear regression. The model was thus rerun with a log-transformation of testosterone, where diagnostics no longer showed any signs of assumption violations.

In order to determine whether gonadectomy altered adult sMRI outcomes, global brain tissue volumes, regional brain tissue volumes, and subcortical volumes at final MRI were modeled as a linear function of condition, correcting for baseline ICV. Global outcomes included total GM (GM), total WM (WM), and total cerebrospinal fluid (CSF). Regional volumes are described in the image analysis section (see above). The Benjamini-Hochberg correction was used to control family-wise error rate due to multiple testing with correlated outcomes. Volumes from left and right hemispheres were combined by region prior to statistical analysis.

In order to determine whether gonadectomy altered the developmental trajectory of intracranial volume, scaled and centered intracranial volume (ICV) was modeled as a function of time, condition, and their interaction using a linear mixed effects model with random intercept, as in the analysis of testosterone. Scaling and centering was achieved by subtracting mean ICV from the individual ICV values and then dividing by the standard deviation of the ICV values. Scaling and centering is recommended when there is heterogeneity in observed values, which can lead to problems with model convergence.

In order to determine whether gonadectomy altered the developmental trajectory of global and regional brain tissue volumes, analyses were performed in two steps: screening and feature selection. This was done to control potential false positives that might arise from testing a large number of regions. In the screening step, all regional and global tissue volumes were scaled and centered, and the following mixed effects model was fit with random intercepts.

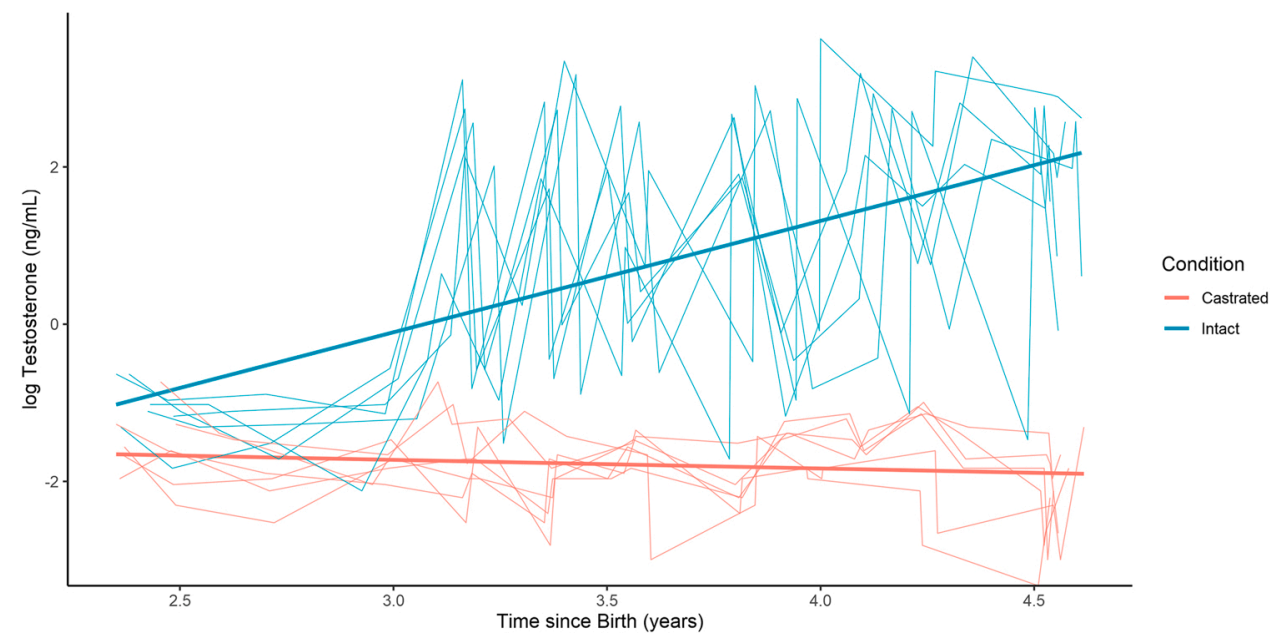

Fig. 1. Log-testosterone trajectories for each monkey with fit lines based on the polynomial functions in Table 1. 
$E[$ Volume $]=\beta_{0 i}+\beta_{1}$ condition $+\beta_{2}$ time $+\beta_{3}$ time $^{2}+\beta_{4}$ condition ${ }^{*}$ time

$$
+\beta_{5} \text { condition }^{*} \text { time }{ }^{2} \text {, }
$$

where time was taken to be the scaled number of days since birth. Eq. 1 was then compared against a random intercept only model using a chisquare test with a Satterthwaite approximation of the degrees of freedom from the lmerTest R package. Quadratic terms were considered to correct for observed non-linearity in the residuals. Regions with model comparisons leading to a significant p-value after BenjaminiHochberg correction for multiple testing were considered to have passed the screening step. This step allows for the evaluation of both time and condition effects on the standardized volumes.

A feature selection step was then performed on the regions which passed the screening step to consider the effect of condition only. For each screened region, the same mixed effects model was fit with random intercepts (Eq. 1). Backwards step-wise selection removed variables from Eq. 1 one at a time, according to their F-test statistics. The model is iteratively refit after removing the variable with the highest p-value until all model variables have a p-value below 0.05 . The model was set up to ensure that lower order age terms (e.g. linear effect of time) could not be removed before higher order terms (e.g. quadratic effect of time). In addition, main effect terms (e.g. condition) could not be removed before interaction terms (e.g. condition*time).

For models with significant condition effects, a permutation test was performed to confirm that the effect was indeed significant. All subjects were reassigned condition status, and the most parsimonious model from the feature selection step for these regions was compared to a reduced model. The reduced model included the same terms as the discovered most parsimonious model, but the terms involving condition were removed. A null distribution of p-values can then be created, and the p-value from the feature selection step is then compared with the pvalues from each of the permutations.

To better understand what effect sizes would be detectable in our sample, we compared Eq. 1 (which includes terms for condition, time, time $^{2}$, condition*time, and condition*time ${ }^{2}$ ) against Eq. 2 (which includes terms for time and time ${ }^{2}$, but no terms related to condition) using a chi squared test.

$E[$ Volume $]=\beta_{0 i}+\beta_{1}$ time $+\beta_{2}$ time ${ }^{2}$

The resulting test statistic captures the overall group effect of the intervention (condition + condition*time + condition $*$ time $^{2}$ ). We then compared each test statistic against the critical value of a chi-square distribution with 3 degrees of freedom and 0.05 level of significance. The resulting values represent the necessary increase in the chi-square statistic required to see a significant effect of condition, condition*time, or condition $*$ time $^{2}$ in our sample. One can also conceptualize this as the proportional increase in Cohen's D necessary to see significance. The values are proportional to the vector of coefficients for the effects of condition, condition*time, and condition*time ${ }^{2}$ together.

All mixed models were fit using the lmer function of the $\mathrm{R}$ package lme4; a seed of 2018 was used, and all tests were evaluated at the 0.05 significance level after correction unless otherwise specified.

Finally, longitudinal standard deviation estimates are reported in Table 6 so that investigators of future longitudinal studies may perform their sample size calculations. These estimates are derived from Fitzmaurice et al. (2012) with modifications for unbalanced and mismatched time points. First, consider the null hypothesis that the effect size of castration is zero where the effect size is the mean difference in the volume. The null hypothesis may be formalized as $H_{0}: \delta^{m, k}=\mu_{1}^{m, k}-$ $\mu_{0}^{m, k}$ where $\delta$ is the effect size, $\mu_{1}^{m, k}$ is the mean type $m$ volume in region $k$ in the castrated group, and $\mu_{0}^{m, k}$ is that in the sham surgery group. We have $n=12$ subjects and each is measured $N_{i}$ times for $i=1, \ldots, n$. Assume the linear model
$Y_{i j}^{m, k}=\beta_{1 i}^{m, k}+\beta_{2 i}^{m, k} t_{i j}+\varepsilon_{i j}^{m, k}$

where $Y_{i j}^{m, k}$ is the matter type $m$ volume of the $k$ th region for subject $i \in$ $1, \ldots, n$ at time $j=0, \ldots N_{i}, t_{i j}$ is the $j$ th time point for subject $i$, and $\varepsilon_{i j} \sim N\left(0, \sigma_{\varepsilon}^{2}\right)$. First, we estimate $\sigma_{\varepsilon}^{2}$ as the average within-subject variance, or $\widehat{\sigma}_{\varepsilon}^{2}=n^{-1} \sum_{i=1}^{n} N_{i}^{-1} \sum_{j=1}^{N_{i}}\left\{Y_{i j}^{m, k}-\bar{Y}_{i j}^{m, k}\right\}^{2}$. Next, we estimate $g_{22}$, the between-subject variance, from fitting the linear mixed effects model in Eqn. 4. Finally, these estimates may be used to find the total variance,

$\sigma_{\beta}^{2}=\operatorname{Var}\left[\widehat{\beta}_{2 i}\right]=\sigma_{\varepsilon}^{2}\left\{\frac{1}{n} \sum_{i=1}^{n} \sum_{j=1}^{N_{i}}\left(t_{i j}-\bar{t}_{i j}\right)^{2}\right\}^{-1}+g_{22}$

and the estimated standard deviation is simply the square-root of Eqn. 5. The estimates found in Table 6 may be used in future analysis according to

$N=\frac{\left\{Z_{(1-\alpha) / 2}+Z_{(1-\gamma)}\right\}^{2} \sigma_{\beta}^{2}}{\pi(1-\pi)\left(\delta^{m, k}\right)^{2}}$,

given some desired power $(1-\gamma) \times 100 \%$ and significance level $\alpha$, where $\gamma, \alpha \in(0,1)$

\section{Results}

Model parameters for log-testosterone values are described in Table 1 , and the effect of the time and condition interaction was found to be significant $(p<0.0001)$, confirming that gonadectomy did eliminate the expected increase in adolescent testosterone as can be seen in Fig. 1 (confirming our earlier analysis reported in Richards et al. (2009)).

All comparisons of adult (final) brain volumes between the gonadectomized and intact groups were found to be non-significant after correction for multiple testing.

The model for developmental change in ICV revealed that there was no significant association with time or condition, and no significant interactions between time and condition (Table 2). Removal of the interaction term led to the same conclusion for the association between ICV and the main effect of condition $(\mathrm{p}=0.4878)$. Individual trajectories of ICV can be seen in Fig. 2.

The screening step revealed that the set of covariates from Eq. 1 had a significant effect $(\mathrm{p}<0.05)$ on $8 \mathrm{WM}$ regions, $6 \mathrm{GM}$ regions, as well as total WM, total GM, and total CSF (see Table 3). Of these regions, only prefrontal GM was found to have a significant effect from the interaction between time and condition in the feature selection step ( $p=0.0475$ for condition $\mathrm{x}$ time and $p=0.0403$ for condition $\mathrm{x}$ time ${ }^{2}$ ) (Table 4). Trajectories can be compared in Fig. 2 (fit lines are based on the polynomial

\section{Table 1}

Model summary for the linear mixed effects model for log-Testosterone, using morning status, time since birth, and condition as covariates with a random intercept.

\begin{tabular}{|c|c|c|c|c|c|}
\hline Effect & Estimate & $\begin{array}{l}\text { Std. } \\
\text { Error }\end{array}$ & $\mathrm{df}$ & p-value & $95 \%$ CI \\
\hline Time of day ${ }^{\dagger}$ & -1.04 & 0.13 & 208.81 & $3.42 \times 10^{-14}$ & $\begin{array}{l}(-1.28 \\
-0.79)\end{array}$ \\
\hline Time (Age) & 4.61 & 0.48 & 209.15 & $<1.00 \times 10^{-20}$ & $\begin{array}{l}(3.66, \\
5.55)\end{array}$ \\
\hline $\begin{array}{l}\text { Condition (Intact } \\
\quad=0 \text { ) }\end{array}$ & 3.01 & 0.68 & 217.80 & $\begin{array}{l}<0.00011 .58 \times \\
10^{-5}\end{array}$ & $\begin{array}{l}(1.69, \\
4.34)\end{array}$ \\
\hline Time ${ }^{*}$ Condition & -5.73 & 0.67 & 209.09 & $\begin{array}{l}<0.00013 .55 \times \\
10^{-15}\end{array}$ & $\begin{array}{l}(-7.04 \\
-4.41)\end{array}$ \\
\hline
\end{tabular}

$\dagger$ Testosterone generally exhibits a diurnal pattern with lower values in the morning and higher values in the evening. Time of day indicates whether the measurement was taken in the morning (time of day $=1$ ) or in the evening (time of day $=0$ ). 
Table 2

Model summary for the linear mixed effects model for standardized intracranial volume, using time and condition as covariates with a random intercept.

\begin{tabular}{|c|c|c|c|c|c|}
\hline Effect & Estimate & $\begin{array}{l}\text { Std. } \\
\text { Error }\end{array}$ & $\mathrm{df}$ & $\begin{array}{l}\text { p- } \\
\text { value }\end{array}$ & $95 \%$ CI \\
\hline Time & 0.07 & 0.21 & 81.00 & 0.7248 & $\begin{array}{l}(-0.34 \\
0.49)\end{array}$ \\
\hline $\begin{array}{l}\text { Condition (Intact }= \\
0 \text { ) }\end{array}$ & 0.63 & 0.65 & 15.40 & 0.3512 & $\begin{array}{l}(-0.63 \\
1.88)\end{array}$ \\
\hline Time*Condition & -0.21 & 0.30 & 81.00 & 0.4786 & $\begin{array}{l}(-0.80 \\
0.37)\end{array}$ \\
\hline
\end{tabular}

functions in Table 4). The permutation test for prefrontal GM had a pvalue of 0.0617

We note that the intact and castrated subjects also exhibit significant differences at the baseline measurement $(p=0.0298)$. However, the effect estimates for the PFC GM volume given in Table 4 suggest that at any given time point, the slope of condition + condition*time + condition time $^{2}$ is positive, signaling less thinning than the intact group, which would have condition + condition $*$ time + condition $*$ time $^{2}=0$. Thus, the trend over time for the castrated group is more positive than that for the intact group, despite the baseline differences. We also note that baseline differences are strongly influenced by animal ZB33, who happens to be quite large. Removal of ZB33 from the analysis attenuates baseline differences, but does not influence the overall trajectory differences (see Supplementary Fig. 2).

The other brain volume variables selected in the screening step were found to have a significant effect of time, or time ${ }^{2}$, but no effect of condition and no interaction between time and condition. Total WM increased across adolescence, while total GM and total CSF decreased. Regionally, WM volumes increased with age in the cerebellum, occipital and parietal lobes, and in temporal auditory and temporal visual cortex. WM volumes decreased in cingulate and PFC. Overall decreases in regional GM volumes were observed in the cerebellum, parietal lobe, and temporal auditory, temporal visual, and cingulate cortices.

Values comparing observed chi-square statistics with the proportional increase necessary to observe significance from the main and interactive effects of condition in a sample of this size can be found in Supplementary Table 1 . Note that the proportional increase varies significantly across different regions and tissue types. For example, in order to observe a significant effect of condition on subcortical GM volume, the $\chi^{2}$ statistic would need to be 55.89 times as large as the one observed. In contrast, the $\chi^{2}$ statistic would only need to be 1.45 times larger than the one observed to achieve significance for condition on frontal GM volume. Supplementary Table 2 presents longitudinal standard deviation estimates for the MRI volumes across all matter types and regions. These numbers can be used to power future studies.

\section{Discussion}

Some of the most salient biological differences between males and females emerge during the pubertal transition when the hypothalamic gonadotropin releasing hormone pulse generator emerges from juvenile suppression, resulting in increasing levels of circulating gonadotropinreleasing hormone $(\mathrm{GnRH})$ and pituitary follicle-stimulating hormone (FSH) and luteinizing hormone (LH), which lead to increases in gonadal
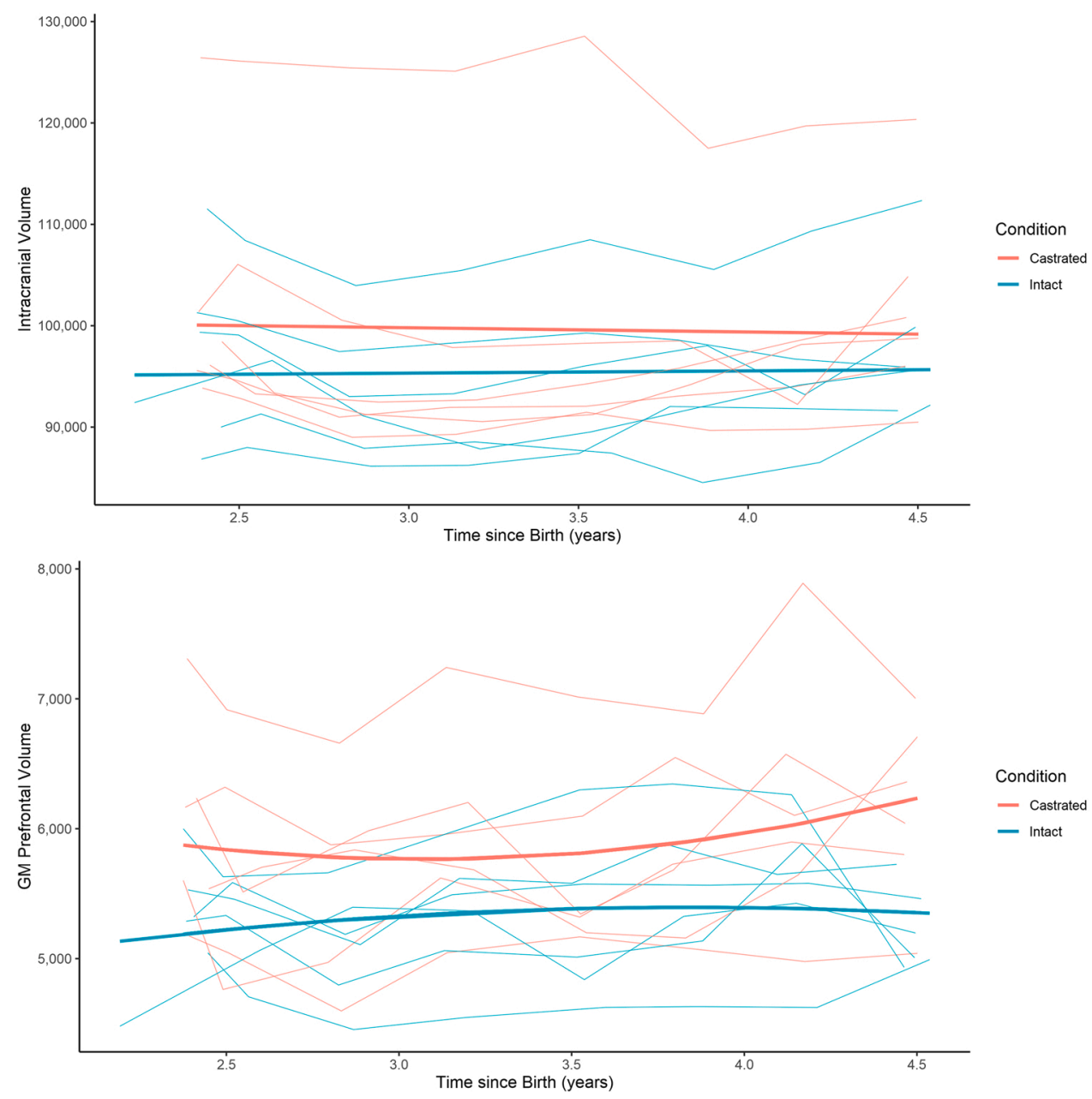

Fig. 2. Observed trajectories for intracranial volume (ICV) and prefrontal GM with fit lines based on the polynomial functions in Table 4 . 
Table 3

Brain regions which were found to have significant model effects under Eq. (1).

\begin{tabular}{llll} 
Matter & Region & Adjusted p-value & $R_{m}^{2}$ \\
\hline \multirow{4}{*}{ Occipital } & $2.88 \times 10^{-2}$ & 0.12 \\
& Temporal Auditory & $6.70 \times 10^{-4}$ & 0.29 \\
& Cerebellum & $6.70 \times 10^{-4}$ & 0.17 \\
& Cingulate & $3.18 \times 10^{-2}$ & 0.07 \\
& Parietal & $2.00 \times 10^{-7}$ & 0.62 \\
& Prefrontal & $1.01 \times 10^{-2}$ & 0.17 \\
& Temporal Visual & $2.93 \times 10^{-7}$ & 0.07 \\
& Pons Medulla & $2.93 \times 10^{-7}$ & 0.16 \\
& Temporal Auditory & $3.45 \times 10^{-2}$ & 0.13 \\
GM & Cerebellum & $1.89 \times 10^{-2}$ & 0.34 \\
& Cingulate & $8.27 \times 10^{-3}$ & 0.86 \\
& Parietal & $6.19 \times 10^{-3}$ & 0.17 \\
& Prefrontal & $2.07 \times 10^{-2}$ & 0.78 \\
& Temporal Visual & $3.23 \times 10^{-5}$ & 0.07 \\
& WM & $3.24 \times 10^{-15}$ & 0.03 \\
& GM & $1.05 \times 10^{-3}$ & 0.89 \\
& CSF & $6.70 \times 10^{-4}$ & 0.01 \\
\hline
\end{tabular}

sex steroids. In humans, this period includes marked increases in the incidence of depression, anxiety, and eating disorders in females (Angold et al., 2003; Hayward and Sanborn, 2002) and increased prevalence of schizophrenia in males (Gotowiec et al., 2003). Sex differences have also been reported in trajectories of global and regional brain volumes during adolescence, measured via sMRI (Aubert-Broche, et al., 2013; Dennison et al., 2013; Lenroot et al., 2007; Mills et al., 2016; Vijayakumar et al., 2016). In this study, we tested whether circulating factors from the male gonad influence adolescent changes in brain morphology in rhesus macaques. We observed a significant effect of gonadectomy on the developmental trajectory of prefrontal gray matter (GM), with intact males showing peak volumes around 3.5 years of age with a subsequent decline. In contrast, prefrontal GM volumes continued to increase in gonadectomized males until the end of the study. This result is in keeping with reports of inverse correlations between circulating testosterone and cortical thickness in dorsolateral PFC of human males between 14 and 22 years of age (Nguyen et al., 2013). We note that final PFC volumes were not larger in gonadectomized animals compared to intact animals. This may be due to inter-individual variation in animal size. A major benefit of longitudinal studies, such as this one, are that they can account for complexity related to individual differences over time. Such effects may be masked in cross-sectional studies (Wolff and Piven, 2014).

We did not observe a significant effect of gonadectomy on prefrontal WM or on any other global or regional brain tissue volumes, though we cannot rule out that effects might be detected in a larger sample. Supplementary Table 1 provides the proportional increase necessary to observe significance from the main and interactive effects of condition in a sample of this size, while Supplementary Table 2 presents longitudinal standard deviation estimates for the MRI volumes across all matter types and regions which can be used to power future studies. Never-theless, the current study suggests that prefrontal GM is more vulnerable to

Table 4

Stepdown step significant effects reduced from Eq. 2. $R_{m}^{2}$ are marginal $R^{2}$ values associated with only fixed effects.

\begin{tabular}{|c|c|c|c|c|c|c|c|}
\hline Matter & Region & $R_{m}^{2}$ & Effect & Estimate & Std. Error & $\mathrm{df}$ & p-value \\
\hline \multirow[b]{6}{*}{ WM } & Occipital & 0.11 & time & 1.58 & 0.42 & 82.05 & $2.70 \times 10^{-4}$ \\
\hline & Temporal Auditory & 0.08 & time & 1.37 & 0.27 & 82.02 & $1.81 \times 10^{-6}$ \\
\hline & Cerebellum & 0.14 & time & 1.83 & 0.36 & 82.06 & $2.85 \times 10^{-6}$ \\
\hline & \multirow[t]{2}{*}{ Cingulate } & 0.05 & time & -1.04 & 0.31 & 82.00 & $1.29 \times 10^{-3}$ \\
\hline & & 0.04 & time & -4.11 & 1.56 & 81.01 & 0.0103 \\
\hline & \multicolumn{2}{|l|}{ Parietal } & time $^{2}$ & 2.55 & 0.79 & 81.01 & $1.85 \times 10^{-3}$ \\
\hline & \multirow{2}{*}{ Prefrontal } & \multirow[t]{2}{*}{0.11} & time & -12.49 & 4.80 & 81.29 & 0.0109 \\
\hline & & & time $^{2}$ & 5.69 & 2.43 & 81.29 & 0.0219 \\
\hline & Temporal Visual & 0.12 & time & 1.70 & 0.23 & 82.01 & $1.27 \times 10^{-10}$ \\
\hline & Pons Medulla & 0.09 & time & 1.47 & 0.21 & 82.01 & $8.09 \times 10^{-10}$ \\
\hline \multirow[t]{14}{*}{ GM } & \multirow[t]{2}{*}{ Temporal Auditory } & \multirow[t]{2}{*}{0.02} & time & -7.04 & 1.97 & 81.02 & $5.90 \times 10^{-4}$ \\
\hline & & & time $^{2}$ & 3.52 & 1.00 & 81.02 & $6.88 \times 10^{-4}$ \\
\hline & Cerebellum & 0.07 & time & -1.26 & 0.32 & 82.02 & $1.83 \times 10^{-4}$ \\
\hline & \multirow[t]{2}{*}{ Cingulate } & \multirow[t]{2}{*}{0.01} & time & -6.02 & 1.52 & 81.01 & $1.54 \times 10^{-4}$ \\
\hline & & & time $^{2}$ & 3.10 & 0.77 & 81.01 & $1.23 \times 10^{-4}$ \\
\hline & \multirow[t]{2}{*}{ Parietal } & \multirow[t]{2}{*}{0.01} & time & -5.54 & 1.39 & 81.01 & $1.51 \times 10^{-4}$ \\
\hline & & & time $^{2}$ & 2.77 & 0.71 & 81.01 & $1.88 \times 10^{-4}$ \\
\hline & \multirow[t]{5}{*}{ Prefrontal $^{\dagger}$} & \multirow[t]{5}{*}{0.18} & condition & 5.76 & 2.60 & 84.03 & 0.0298 \\
\hline & & & time & 3.73 & 3.74 & 70.03 & 0.322 \\
\hline & & & time $^{2}$ & -1.68 & 1.90 & 79.03 & 0.379 \\
\hline & & & condition*time & -10.85 & 5.39 & 79.03 & 0.0475 \\
\hline & & & condition*time ${ }^{2}$ & 5.69 & 2.73 & 79.03 & 0.0403 \\
\hline & \multirow[t]{2}{*}{ Temporal Visual } & \multirow[t]{2}{*}{0.05} & time & -9.63 & 2.30 & 81.03 & $7.26 \times 10^{-5}$ \\
\hline & & & time $^{2}$ & 4.47 & 1.17 & 81.03 & $2.51 \times 10^{-4}$ \\
\hline \multirow[t]{7}{*}{ Total } & \multirow[t]{2}{*}{ WM } & \multirow[t]{2}{*}{0.09} & time & -4.31 & 1.45 & 81.01 & $3.99 \times 10^{-3}$ \\
\hline & & & time $^{2}$ & 2.88 & 0.74 & 81.01 & $1.90 \times 10^{-4}$ \\
\hline & \multirow[t]{3}{*}{ GM } & \multirow[t]{3}{*}{0.01} & & & & & \\
\hline & & & time & -6.34 & 1.52 & 81.01 & $7.32 \times 10^{-5}$ \\
\hline & & & time $^{2}$ & 3.13 & 0.77 & 81.01 & $1.08 \times 10^{-4}$ \\
\hline & \multirow[t]{2}{*}{ CSF } & \multirow[t]{2}{*}{0.11} & time & -10.56 & 3.70 & 81.10 & $5.48 \times 10^{-3}$ \\
\hline & & & time $^{2}$ & 4.66 & 1.88 & 81.10 & 0.0151 \\
\hline
\end{tabular}

\footnotetext{
$\dagger$ model where condition was significant.
} 
gonadectomy than other regions. This is particularly interesting as this region's functional correlates include high-level cognitive processes such as working memory, social cognition, and abstract thought. The primate PFC is also evolutionarily distinct in being hyperscaled in comparison to isocortex and whole brain. The PFC shares extensive interconnections with subcortical regions, sensorimotor cortices, and other association cortices (Teffer and Semendeferi, 2012). Consequently, disrupted PFC development could have wide-ranging functional effects and mayexplain behavioral changes previously reported in these monkeys, which include higher levels of prepulse inhibition and altered responses to social stimuli (Allen et al., 2014; Morris et al., 2010; Richards et al., 2009).

The PFC matures relatively late in comparison with the rest of the brain at both the structural and neurochemical level (Caballero et al., 2016). This prolonged maturational trajectory may contribute to its particular vulnerability to gonadectomy, although many other brain regions showed significant time-related changes, including cerebellum, parietal lobe, and temporal auditory, temporal visual, and cingulate cortices. Other areas such as pons/medulla, frontal lobe (excluding prefrontal areas), and temporal limbic and insular cortex showed little change during adolescence and may represent regions that are essentially mature prior to puberty and thus were unlikely to be affected by peripubertal gonadectomy. The vulnerability of PFC may also reflect regional differences in distribution of hormone receptors and enzymes which process testosterone, such as aromatase and 5-alpha reductase. High-affinity androgen-binging sites appear to be widely distributed in the brain of mature rhesus macaques, and production of 5-alpha reduced androgens is also widespread. In contrast, production of estrogen via aromatase appears to be more restricted, occurring primarily in subcortical areas and, notably, in PFC (Clark et al., 1988). We also note that the molecular mechanisms which produce sexually-dimorphic neuroanatomical endpoints vary significantly from region to region (McCarthy et al., 2015). For example, the prostaglandin PGE2 plays an important role in sexual differentiation of the medial preoptic area (Amateau and McCarthy, 2004), while PI3 and MAP kinases play a key role in sexual differentiation of the hypothalamus (Schwarz etal., 2008). Some of these regional mechanisms may be more robust to perturbations, such as gonadectomy, than others.

We note that in human beings, PFC thinning during adolescence is accompanied by a marked increase in PFC (and other cortical) WM, with the rate of increase being greater in males (Schulz and Sisk, 2017). Consequently, one might hypothesize that pubertal testosterone induces a switch in PFC development (promoting both GM thinning and WM growth). We did not observe effects of gonadectomy on WM in the PFC or elsewhere. Thus, our results do not support this model in its entirety, though they are compatible with the hypothesis that testosterone promotes a switch in GM development from thickening to thinning. Furthermore, we did not observe sharp increases in PFC WM development during adolescence in this study - instead we observed decreases in PFC WM (See Supplementary Figure 3), which mirror an earlier cross-sectional study of regional GM and WM development in macaque (Knickmeyer et al., 2010). The absence of gonadectomy effects on WM development may appear surprising, given reports that the androgen receptor influences gene expression of myelin proteins in the peripheral nervous system (Melcangi et al., 2003) and testosterone plays a role in the spontaneous regeneration of myelin in the central nervous system (Bielecki et al., 2016). As before, we note that significant effects might be observed in a larger sample. We also note that the imaging technique used in this study does not directly index myelination. Future studies using magnetization transfer (MT) or myelin water imaging (MWI) could address this question more directly. Furthermore, the impact of testosterone on brain cells in vivo is dependent on multiple variables including the age of exposure, duration of exposure, and prior history of exposure (Schulz and Sisk, 2016; Sinclair et al., 2014). Consequently, we cannot rule out the possibility that gonadectomy would impact WM in a different context than the one studied here. However, our results are consistent with several prior studies in rats that suggest ovarian hormones, rather than testicular hormones, influence white matter development during adolescence, thereby producing adult sex-differences. The first study revealed that prepubertal gonadectomy did not influence WM volumes in the medial prefrontal cortex (mPFC) of males, but did influence WM volumes in females (Koss et al., 2015). The second demonstrated that prepubertal gonadectomy led to a significant reduction of myelin basic protein (MBP) in the orbitofrontal cortex of adult females, but did not affect MBP levels in adult males (Darling and Daniel, 2019).

Non-significant results for other brain regions must be taken with certain caveats. First, individual variation in regional volumes from monkey to monkey and/or variation arising due to measurement noise could have obscured relatively subtle effects of gonadal steroids. This effect may be more pronounced in some regions than others. Although the current study found insufficient evidence, a larger study might reveal subtle effects of gonadectomy in other regions. Second, gonadal steroids may influence neurodevelopmental processes occurring at a resolution not captured by our sMRI protocol. Improved acquisition sequences or use of higher-strength scanners would allow more detailed assessments of neuroanatomy, including direct measures of surface area and cortical thickness. Third, gonadectomy may have detectable effects on imaging modalities not included in the current study, such as diffusion tensor imaging or functional activity/connectivity. Finally, gonadal steroids may exert more widespread effects in human beings than they do in macaques. We think this final possibility is somewhat unlikely given the phylogenetic closeness between humans and macaques as well as similarities in pubertal physiology and brain development (Hodges and Palmert, 2007; Knickmeyer et al., 2010; Scott et al., 2016). As regards pubertal physiology, like humans, juvenile rhesus macaques experience a protracted period of gonadal quiescence followed by a steep increase in gonadal steroid production that continues over many years. This is in contrast to rodents where the transition to puberty is more gradual and continuous, but occurs over weeks (Hodges and Palmert, 2007). However, unlike humans, rhesus macaques are seasonal breeders and undergo significant changes in sex hormones during the year (increasing during the autumn months in the Northern Hemisphere). Thus, the increase in sex steroids can be sharp but variable. As regards brain development, human males exhibit a greater rate of change in brain tissue volumes across adolescence compared to females (Lenroot et al., 2007), an effect which is particularly marked for WM (Aubert-Broche et al., 2013). This finding of prolonged maturation in males has also been observed in macaques (Konigsberg et al., 1990; Scott et al., 2016). Although the current study did not include a female comparison group, both intact and gonadectomized males showed protracted increases in WM and protracted decreases in parietal and temporal GM across adolescence, potentially continuing into adulthood, patterns also evident in humans (Mills et al., 2016).

Strengths of the current study include the direct manipulation of adolescent sex steroid levels via gonadectomy and acquisition of longitudinal neuroimaging data throughout adolescence into young adulthood. There are also several limitations. First, the study lacks a female control group and a gonadectomy + testosterone replacement group. Future studies should include intact and oophorectomized females as well as intact and gonadectomized males and incorporate hormone replacement arms to delineate effects of different gonadal hormones. Future studies might also involve direct manipulation of adrenarche as well as gonadarche, as this constitutes another component of puberty (Fechner, 2003). Finally, we note that gonadal hormones, including testosterone and estrogen, participate in dynamic hormonal networks. Individual responses to gonadectomy may be modulated by other factors such as genetic variation in androgen and estrogen receptors, and in enzymes responsible for interconversion and metabolism (Xia et al., 2014). Genetic variation in the estrogen receptor is associated with neonatal brain volume (Knickmeyer et al., 2014), and testosterone-related increases in white-matter volumes of male 
adolescents are influenced by the number of CAG triplets in exon 1 of the androgen receptorgene ( $A R$ ) (Perrin et al., 2008), which is thought to be inversely proportional to AR transcriptional activity (Chang, 2002).

Despite these limitations, the current study provides important new information about the role of gonadal steroids in adolescent brain development. In particular, our finding that gonadectomy influences GM development in the PFC may have important implications for understanding the role of pubertal sex steroids in shaping developmental changes in cognitive control, working memory, emotional regulation, and vulnerability to psychopathology.

\section{Author contributions}

Rebecca C. Knickmeyer (Methodology, Supervision, Writing-original draft, Writing-review and editing) Crystal T. Nguyen (Methodology, Formal Analysis, Visualization, Writing-original draft, Writing - review \& editing.), Jeffrey T. Young (Formal analysis, Supervision, Writingreview \& editing), Anne Haunton (Formal analysis, Writing-review \& editing), Michael R. Kosorok (Methodology, Formal Analysis, Supervision, Writing-review \& editing), John H. Gilmore (Methodology, Writing-review \& editing), Martin Styner (Funding acquisition, Methodology, Supervision, Software, Writing-review \& editing), Debora A. Rothmond (Data curation, Investigation, Project administration, Writing-review \& editing), Pamela L. Noble (Investigation, Methodology, Writing-review \& editing), Rhoshel Lenroot (Investigation, Methodology, Writing-review \& editing), and Cynthia Shannon Weickert (Conceptualization, Funding acquisition, Investigation, Supervision, Project administration, Writing-review \& editing)

\section{Data statement}

All data is available upon request to the authors.

\section{Declaration of Competing Interest}

None.

\section{Acknowledgments}

This work was funded by the National Institute of Mental Health, Intramural Program (IRP), the National Institutes of Health (MH901645, MH100031, HD079124), and the NSW Ministry of Health, Office of Health and Medical Research. CSW is also recipient of a National Health and Medical Research Council (Australia) Principal Research Fellowship (PRF)(\#1117079). We thank the staff of the UNC Neuro Image Research and Analysis Laboratories, especially Joseph Blocher and Yundi Wendy Shi, and our student volunteers including Camille Haywood, Elizabeth Kennedy, Ian Funk, Yasmeen Mansour, and Shelby Rawlins. We would also like to thank Dr. A. Brent Richards, Sarah Ward, Debbie Delaney Greene, and Erin Hanson Burkes for their expertise and assistance with the extensive physiological and behavioral testing throughout the study. We are grateful for the tireless efforts of Dr. Ruth A. Woodward and all of the veterinary and animal care staff of the NIH animal facility in Poolesville and NIMH Primate Core.

\section{Appendix A. Supplementary data}

Supplementary material related to this article can be found, in the online version, at doi:https://doi.org/10.1016/j.psyneuen.2020.1050 68.

\section{References}

Ahmed, E.I., Zehr, J.L., Schulz, K.M., Lorenz, B.H., DonCarlos, L.L., Sisk, C.L., 2008. Pubertal hormones modulate the addition of new cells to sexually dimorphic brain regions. Nat. Neurosci. 11, 995-997.
Allen, K.M., Fung, S.J., Rothmond, D.A., Noble, P.L., Weickert, C.S., 2014. Gonadectomy increases neurogenesis in the male adolescent rhesus macaque hippocampus. Hippocampus 24, 225-238.

Amateau, S.K., McCarthy, M.M., 2004. Induction of PGE(2) by estradiol mediates developmental masculinization of sex behavior. Nat. Neurosci. 7, 643-650.

Angold, A., Worthman, C.M., Costello, E.J., 2003. Puberty and depression. In: Hayward, C. (Ed.), Gender Differences at Puberty. Cambridge University Press, Cambridge, pp. 137-164.

Aubert-Broche, B., Fonov, V.S., Garcia-Lorenzo, D., Mouiha, A., Guizard, N., Coupe, P., Eskildsen, S.F., Collins, D.L., 2013. A new method for structural volume analysis of longitudinal brain MRI data and its application in studying the growth trajectories of anatomical brain structures in childhood. Neuroimage 82, 393-402.

Bielecki, B., Mattern, C., Ghoumari, A.M., Javaid, S., Smietanka, K., Abi Ghanem, C., Mhaouty-Kodja, S., Ghandour, M.S., Baulieu, E.E., Franklin, R.J., Schumacher, M., Traiffort, E., 2016. Unexpected central role of the androgen receptor in the spontaneous regeneration of myelin. Proc. Natl. Acad. Sci. U. S. A. 113, 14829-14834.

Borrell, V., Calegari, F., 2014. Mechanisms of brain evolution: regulation of neural progenitor cell diversity and cell cycle length. Neurosci. Res. 86, 14-24.

Bowen, W.H., Koch, G., 1970. Determination of age in monkeys (Macaca irus) on the basis of dental development. Lab. Anim. 4, 113-124.

Bramen, J.E., Hranilovich, J.A., Dahl, R.E., Forbes, E.E., Chen, J., Toga, A.W., Dinov, I.D., Worthman, C.M., Sowell, E.R., 2011. Puberty influences medial temporal lobe and cortical gray matter maturation differently in boys than girls matched for sexual maturity. Cereb. Cortex 21, 636-646.

Breedlove, S.M., Jordan, C.L., Kelley, D.B., 2002. What neuromuscular systems tell us about hormones and behavior. In: Pfaff, D., Arnold, A., Etgen, A., Fahrbach, S., Rubin, R. (Eds.), Hormones, Brain and Behavior. Academic Press, New York, pp. 193-221.

Brenhouse, H.C., Andersen, S.L., 2011. Developmental trajectories during adolescence in males and females: a cross-species understanding of underlying brain changes. Neurosci. Biobehav. Rev. 35, 1687-1703.

Caballero, A., Granberg, R., Tseng, K.Y., 2016. Mechanisms contributing to prefrontal cortex maturation during adolescence. Neurosci. Biobehav. RSev. 70, 4-12.

Chang, C., 2002. Androgens and Androgen Receptor: Mechanisms, Functions, and Clinical Application. Kluwer Academic Publishers, Boston.

Clark, A.S., Maclusky, N.J., Goldmanrakic, P.S., 1988. Androgen binding and metabolism in the cerebral-cortex of the developing rhesus-monkey. Endocrinology 123, 932-940.

Darling, J.S., Daniel, J.M., 2019. Pubertal hormones mediate sex differences in levels of myelin basic protein in the orbitofrontal cortex of adult rats. Neuroscience 406, 487-495.

De Lorme, K.C., Schulz, K.M., Salas-Ramirez, K.Y., Sisk, C.L., 2012. Pubertal testosterone organizes regional volume and neuronal number within the medial amygdala of adult male Syrian hamsters. Brain Res. 1460, 33-40.

Dennison, M., Whittle, S., Yucel, M., Vijayakumar, N., Kline, A., Simmons, J., Allen, N.B., 2013. Mapping subcortical brain maturation during adolescence: evidence of hemisphere- and sex-specific longitudinal changes. Dev. Sci. 16, 772-791.

Fechner, P.Y., 2003. The biology of puberty: new developments in sex differences. In: Hayward, C. (Ed.), Gender Differences at Puberty. Cambridge University Press, Cambridge, pp. 17-28.

Fitzmaurice, G.M., Laird, N.M., Ware, J.H., 2012. Applied Longitudinal Analysis. John Wiley \& Sons, Hoboken.

Giedd, J.N., 2004. Structural magnetic resonance imaging of the adolescent brain. Ann. N. Y. Acad. Sci. 1021, 77-85.

Giedd, J.N., Blumenthal, J., Jeffries, N.O., Castellanos, F.X., Liu, H., Zijdenbos, A. Paus, T., Evans, A.C., Rapoport, J.L., 1999. Brain development during childhood and adolescence: a longitudinal MRI study. Nat. Neurosci. 2, 861-863.

Gotowiec, A., Seeman, M.V., Cohen, R.Z., 2003. Puberty and schizophrenia. In: Hayward, C. (Ed.), Gender Differences at Puberty. Cambridge University Press, Cambridge, pp. 165-186.

Hayward, C., Sanborn, K., 2002. Puberty and the emergence of gender differences in psychopathology. J. Adolesc. Health 30, 49-58.

Herting, M.M., Gautam, P., Spielberg, J.M., Kan, E., Dahl, R.E., Sowell, E.R., 2014. The role of testosterone and estradiol in brain volume changes across adolescence: a longitudinal structural MRI study. Hum. Brain Mapp. 35, 5633-5645.

Hodges, C.A., Palmert, M.R., 2007. Genetic regulation of the variation in pubertal timing. In: Pescovitz, O.H., Walvoord, E.C. (Eds.), When Puberty Is Precocious: Scientific and Clinical Aspects. Humana Press, Totowa, New Jersey, pp. 83-104.

Kaczkurkin, A.N., Raznahan, A., Satterthwaite, T.D., 2019. Sex differences in the developing brain: insights from multimodal neuroimaging. Neuropsychopharmacology 44, 71-85.

Knickmeyer, R.C., Styner, M., Short, S.J., Lubach, G.R., Kang, C., Hamer, R., Coe, C.L., Gilmore, J.H., 2010. Maturational trajectories of cortical brain development through the pubertal transition: unique species and sex differences in the monkey revealed through structural magnetic resonance imaging. Cereb. Cortex 20, 1053-1063.

Knickmeyer, R.C., Wang, J., Zhu, H., Geng, X., Woolson, S., Hamer, R.M., Konneker, T., Lin, W., Styner, M., Gilmore, J.H., 2014. Common variants in psychiatric risk genes predict brain structure at birth. Cereb. Cortex 24, 1230-1246.

Konigsberg, L., Falk, D., Hildebolt, C., Vannier, M., Cheverud, J., Helmkamp, R.C., 1990. External brain morphology in Rhesus macaques (Macaca-Mulatta). J. Hum. Evol. 19, 269-284.

Koss, W.A., Lloyd, M.M., Sadowski, R.N., Wise, L.M., Juraska, J.M., 2015. Gonadectomy before puberty increases the number of neurons and glia in the medial prefrontal cortex of female, but not male. Rats. Dev. Psychobiol. 57, 305-312. 
Lenroot, R.K., Gogtay, N., Greenstein, D.K., Wells, E.M., Wallace, G.L., Clasen, L.S.,

Blumenthal, J.D., Lerch, J., Zijdenbos, A.P., Evans, A.C., Thompson, P.M., Giedd, J. N., 2007. Sexual dimorphism of brain developmental trajectories during childhood and adolescence. Neuroimage 36, 1065-1073.

McCarthy, M.M., Pickett, L.A., VanRyzin, J.W., Kight, K.E., 2015. Surprising origins of sex differences in the brain. Horm. Behav. 76, 3-10.

McEwen, B.S., Milner, T.A., 2017. Understanding the broad influence of sex hormones and sex differences in the brain. J. Neurosci. Res. 95, 24-39.

McEwen, B.S., Akama, K.T., Spencer-Segal, J.L., Milner, T.A., Waters, E.M., 2012. Estrogen effects on the brain: actions beyond the hypothalamus via novel mechanisms. Behav. Neurosci. 126, 4-16.

Melcangi, R.C., Ballabio, M., Cavarretta, I., Gonzalez, L.C., Leonelli, E., Veiga, S., Martini, L., Magnaghi, V., 2003. Effects of neuroactive steroids on myelin of peripheral nervous system. J. Steroid Biochem. Mol. Biol. 85, 323-327.

Mendle, J., 2014. Why puberty matters for psychopathology. Child Dev. Perspect. 8, 218-222.

Mills, K.L., Goddings, A.L., Herting, M.M., Meuwese, R., Blakemore, S.J., Crone, E.A., Dahl, R.E., Guroglu, B., Raznahan, A., Sowell, E.R., Tamnes, C.K., 2016. Structural brain development between childhood and adulthood: convergence across four longitudinal samples. Neuroimage 141, 273-281.

Morishita, M., Maejima, S., Tsukahara, S., 2017. Gonadal hormone-dependent sexua differentiation of a female-biased sexually dimorphic cell group in the principal nucleus of the bed nucleus of the stria terminalis in mice. Endocrinology 158, 3512-3525.

Morris, R.W., Fung, S.J., Rothmond, D.A., Richards, B., Ward, S., Noble, P.L., Woodward, R.A., Weickert, C.S., Winslow, J.T., 2010. The effect of gonadectomy on prepulse inhibition and fear-potentiated startle in adolescent rhesus macaques. Psychoneuroendocrinology 35, 896-905.

Neufang, S., Specht, K., Hausmann, M., Gunturkun, O., Herpertz-Dahlmann, B., Fink, G. R., Konrad, K., 2009. Sex differences and the impact of steroid hormones on the developing human brain. Cereb. Cortex 19, 464-473.

Nguyen, T.V., McCracken, J., Ducharme, S., Botteron, K.N., Mahabir, M., Johnson, W., Israel, M., Evans, A.C., Karama, S., 2013. Testosterone-related cortical maturation across childhood and adolescence. Cereb. Cortex 23, 1424-1432.

Nunez, J.L., Lauschke, D.M., Juraska, J.M., 2001. Cell death in the development of the posterior cortex in male and female rats. J. Comp. Neurol. 436, 32-41.

Nunez, J.L., Sodhi, J., Juraska, J.M., 2002. Ovarian hormones after postnatal day 20 reduce neuron number in the rat primary visual cortex. J. Neurobiol. 52, 312-321.

Peper, J.S., Brouwer, R.M., Schnack, H.G., van Baal, G.C., van Leeuwen, M., van den Berg, S.M., Delemarre-Van de Waal, H.A., Boomsma, D.I., Kahn, R.S., Hulshoff Pol, H.E., 2009. Sex steroids and brain structure in pubertal boys and girls. Psychoneuroendocrinology 34, 332-342.

Perrin, J.S., Herve, P.Y., Leonard, G., Perron, M., Pike, G.B., Pitiot, A., Richer, L., Veillette, S., Pausova, Z., Paus, T., 2008. Growth of white matter in the adolescent brain: role of testosterone and androgen receptor. J. Neurosci. 28, 9519-9524.

Raznahan, A., Shaw, P., Lalonde, F., Stockman, M., Wallace, G.L., Greenstein, D., Clasen, L., Gogtay, N., Giedd, J.N., 2011. How does your cortex grow? J. Neurosci. 31, 7174-7177.

Reuter, M., Fischl, B., 2011. Avoiding asymmetry-induced bias in longitudinal image processing. Neuroimage 57, 19-21.

Richards, A.B., Morris, R.W., Ward, S., Schmitz, S., Rothmond, D.A., Noble, P.L Woodward, R.A., Winslow, J.T., Weickert, C.S., 2009. Gonadectomy negatively impacts social behavior of adolescent male primates. Horm. Behav. 56, 140-148.
Romeo, R.D., Staub, D., Jasnow, A.M., Karatsoreos, I.N., Thornton, J.E., McEwen, B.S., 2005. Dihydrotestosterone increases hippocampal N-methyl-D-aspartate binding but does not affect choline acetyltransferase cell number in the forebrain or choline transporter levels in the CA1 region of adult male rats. Endocrinology 146, 2091-2097.

Satterthwaite, T.D., Vandekar, S., Wolf, D.H., Ruparel, K., Roalf, D.R., Jackson, C., Elliott, M.A., Bilker, W.B., Calkins, M.E., Prabhakaran, K., Davatzikos, C., Hakonarson, H., Gur, R.E., Gur, R.C., 2014. Sex differences in the effect of puberty on hippocampal morphology. J. Am. Acad. Child Adolesc. Psychiatry 53, 341-350.

Schulz, K.M., Sisk, C.L., 2016. The organizing actions of adolescent gonadal steroid hormones on brain and behavioral development. Neurosci. Biobehav. Rev. 70, $148-158$.

Schulz, K.M., Sisk, C.L., 2017. Gonadal Hormonal Influences on the Adolescent Brain and Trajectories of Behavioral Development, Hormones, Brain and Behavior (Third Edition). Elsevier, pp. 293-307.

Schwarz, J.M., Liang, S.L., Thompson, S.M., McCarthy, M.M., 2008. Estradiol induces hypothalamic dendritic spines by enhancing glutamate release: a mechanism for organizational sex differences. Neuron 58, 584-598.

Scott, J.A., Grayson, D., Fletcher, E., Lee, A., Bauman, M.D., Schumann, C.M., Buonocore, M.H., Amaral, D.G., 2016. Longitudinal analysis of the developing rhesus monkey brain using magnetic resonance imaging: birth to adulthood. Brain Struct. Funct. 221, 2847-2871.

Shi, Y., Budin, F., Yapuncich, E., Rumple, A., Young, J.T., Payne, C., Zhang, X., Hu, X., Godfrey, J., Howell, B., Sanchez, M.M., Styner, M.A., 2016. UNC-emory infant atlases for macaque brain image analysis: postnatal brain development through 12 months. Front. Neurosci. 10, 617.

Sinclair, D., Purves-Tyson, T.D., Allen, K.M., Weickert, C.S., 2014. Impacts of stress and sex hormones on dopamine neurotransmission in the adolescent brain. Psychopharmacology (Berl.) 231, 1581-1599.

Tamnes, C.K., Walhovd, K.B., Dale, A.M., Ostby, Y., Grydeland, H., Richardson, G., Westlye, L.T., Roddey, J.C., Hagler Jr., D.J., Due-Tonnessen, P., Holland, D., Fjell, A. M., 2013. Brain development and aging: overlapping and unique patterns of change. Neuroimage 68, 63-74.

Teffer, K., Semendeferi, K., 2012. Human prefrontal cortex: evolution, development, and pathology. Prog. Brain Res. 195, 191-218.

Vijayakumar, N., Allen, N.B., Youssef, G., Dennison, M., Yucel, M., Simmons, J.G., Whittle, S., 2016. Brain development during adolescence: a mixed-longitudinal investigation of cortical thickness, surface area, and volume. Hum. Brain Mapp. 37, 2027-2038.

Wang, J., Vachet, C., Rumple, A., Gouttard, S., Ouziel, C., Perrot, E., Du, G., Huang, X., Gerig, G., Styner, M., 2014. Multi-atlas segmentation of subcortical brain structures via the AutoSeg software pipeline. Front. Neuroinform. 8, 7.

Wolff, J.J., Piven, J., 2014. Neurodevelopmental disorders: accelerating progress in autism through developmental research. Nat. Rev. Neurol. 10, 431-432.

Xia, K., Yu, Y., Ahn, M., Zhu, H., Zou, F., Gilmore, J.H., Knickmeyer, R.C., 2014. Environmental and genetic contributors to salivary testosterone levels in males. Front. Endocrinol. (Lausanne).

Young, J.T., Shi, Y., Niethammer, M., Grauer, M., Coe, C.L., Lubach, G.R., Davis, B., Budin, F., Knickmeyer, R.C., Alexander, A.L., Styner, M.A., 2017. The UNCWisconsin Rhesus macaque neurodevelopment database: a structural MRI and DTI database of early postnatal development. Front. Neurosci. 11, 29. 